\title{
RELAÇÃO ENTRE JUDICIALIZAÇÃO E FATORES SOCIOECONÔMICOS E DE DESEMPENHO DO SISTEMA DE SAÚDE
}

\author{
Relationship between the judicialization and socioeconomic and performance- \\ related factors of the health system
}

\section{Relación entre judicialización y factores socioeconómicos y de desempeño del sistema de salud}

Rafael Lutzoff de Camargo Vaz

Universidade de São Paulo - USP - Ribeirão Preto (SP) - Brasil

Matheus da Costa Gomes (OrclD)

Universidade de São Paulo - USP - Ribeirão Preto (SP) - Brasil

Jessica Adrielle Teixeira Santos (OrcID)

Universidade de São Paulo - USP - Ribeirão Preto (SP) - Brasil

Carlos Alberto Grespan Bonacim (OrcID)

Universidade de São Paulo - USP - Ribeirão Preto (SP) - Brasil

\section{RESUMO}

Objetivo: Analisar a relação de fatores socioeconômicos e de desempenho do sistema de saúde com a ocorrência de processos judiciais contra o setor público da saúde no Brasil. Métodos: Trata-se de um estudo transversal, realizado entre 2015 e 2016 , com dados secundários de 641 municípios do estado de São Paulo, Brasil. A análise ocorreu por meio do Índice de Gini e do Índice de Desempenho do Sistema Único de Saúde (IDSUS), além do número de processos judiciais sobre saúde, indicador da judicialização. As variáveis utilizadas correspondem ao ano de 2010, devido à abrangência e à qualidade dos indicadores que compõem o IDSUS desse ano. Analisaram-se os dados mediante estatística descritiva, matriz de correlação de Pearson e regressão linear múltipla. Resultados: Verificou-se maior incidência de ações judiciais sobre grandes polos urbanos: o DRS I Grande São Paulo obteve o maior número (35,5\%,; n=8.189), seguido pelo DRS XV - São José Do Rio Preto (15,9\%; n=3.679). A maioria dos municípios do estado de São Paulo está concentrada no Grupo Homogêneo 5 do IDSUS, sem estrutura para atendimentos especializados $(62,8 \%$; $n=405)$, sendo que somente $0,9 \%$ dos municípios pertencem ao grupo 1 ( $n=6)$, com melhores condições de atendimento. Como principal resultado, encontrou-se relação linear positiva entre o IDSUS e o número de ações judiciais $(p<0,05)$. Conclusão: Não houve relação linear significativa dos fatores socioeconômicos analisados com a ocorrência de ações judiciais contra o setor público da saúde, contudo verificou-se uma maior judicialização em localidades com melhor desempenho dos serviços de saúde (IDSUS).

Descritores: Judicialização da Saúde; Poder Judiciário; Política Pública; Saúde Pública.

\section{ABSTRACT}

Objective: To analyze the relationship between socioeconomic and performance-related factors of the health system and the incidence of lawsuits against the public health sector in Brazil. Methods: This is a cross-sectional study conducted between 2015 and 2016 with secondary data from 641 municipalities in the State of São Paulo, Brazil. The analysis was performed through the Gini Index and the Performance Index of the Unified Health System (Índice de Desempenho do Sistema Único de Saúde - IDSUS), in addition to the number of lawsuits against the public health sector, indicator of the judicialization of health. The variables used correspond to the year 2010 because of the breadth and quality of the indicators forming the IDSUS that year. Data was analyzed using descriptive statistics, Pearson's correlation matrix and multiple linear regression. Results: There was a higher incidence of lawsuits in large urban centers: DRS I - Metropolitan São Paulo obtained the highest number (35.5\%, $n=8,189)$, followed by DRS XV - São José do Rio Preto $(15.9 \%, n=3,679)$. Most of the municipalities in the State of São Paulo are concentrated in the Homogeneous Group 5 of IDSUS, lacking structure for specialized care $(62.8 \%, n=405$, and only $0.9 \%$ of the municipalities belong to Group $1(n=6)$, with better conditions for health care delivery. As a main result, a positive linear relationship was found between 
IDSUS and the number of lawsuits ( $p<0.05)$. Conclusion: There was no significant linear relation between the socioeconomic factors analyzed and the incidence of lawsuits against the public health sector; however, greater judicialization was observed in places with better performance of health services (IDSUS).

Descriptors: Judicialization of Health; Judiciary; Public Policy; Public Health.

\section{RESUMEN}

Objetivo: Analizar la relación entre los factores socioeconómicos y de desempeño del sistema de salud con la ocurrencia de procesos judiciales contra el sector público de la salud de Brasil. Métodos: Se trata de un estudio transversal realizado entre 2015 y 2016 con datos secundarios de 641 municipios del estado de São Paulo, Brasil. El análisis se dio a través del Índice de Gini y del Índice de Desempeño del Sistema Único de Salud (IDSUS) además del número de procesos judiciales sobre salud, el indicador de la judicialización. Las variables utilizadas corresponden al año 2010 por la amplitud y la calidad de los indicadores que componen el IDSUS de ese año. Se analizaron los datos con la estadística descriptiva, la matriz de correlación de Pearson y la regresión linear múltiple. Resultados: Se ha verificado mayor incidencia de acciones judiciales sobre los grandes polos urbanos: el DRS I - Grande São Paulo obtuvo mayor número (35,5\%; $n=8.189)$, seguido del DRS XV - São José de Rio Preto (15,9\%; n=3.679). La mayoría de los municipios del estado de São Paulo está concentrada en el Grupo Homogéneo 5 del IDSUS, sin estructura para la atención especializada $(62,8 \% ; n=405)$ y solamente el $0,9 \%$ de los municipios pertenecen al grupo 1 ( $n=6)$, con mejores condiciones de asistencia. Se encontró relación linear positiva entre el IDSUS y el número de acciones judiciales $(p<0,05)$ como resultado principal. Conclusión: No hubo relación linear significativa de los factores socioeconómicos analizados con la ocurrencia de acciones judiciales contra el sector público de la salud, sin embargo, se ha verificado mayor judicialización en los sitios con mejor desempeño de los servicios de salud (IDSUS).

Descriptores: Judicialización de la Salud; Poder Judicial; Política Pública; Salud Pública.

\section{INTRODUÇÃO}

No contexto brasileiro, um dos principais desafios para a gestão pública é garantir à população uma assistência em saúde de qualidade apesar da escassez de recursos financeiros ${ }^{(1-5)}$. Neste ínterim, o direito fundamental à saúde vem sendo concretizado por meio de decisões judiciais que impõem ao poder público a obrigação de fornecer insumos, equipamentos e cirurgias para os cidadãos que não estão tendo seus direitos completamente atendidos pelo Estado brasileiro. Este fenômeno é denominado de "judicialização da saúde", ou seja, a intervenção do setor judiciário para obtenção de medicamentos ou tratamentos não contemplados pelo Sistema Único de Saúde (SUS) ${ }^{(6,7)}$.

Estima-se que, no ano de 2010, os gastos com demandas judiciais somaram o equivalente a quase $2 \%$ do orçamento total da Saúde ${ }^{(8)}$. De acordo com uma análise realizada pela Advocacia Geral da União, evidenciou-se, no ano 2012 , um crescimento de quase $25 \%$ no número de processos judiciais solicitando serviços ou insumos dessa natureza em relação ao ano de $2009^{(9)}$. Segundo dados do Ministério da Saúde, os gastos da União com saúde por ordem judicial aumentaram 500\% em apenas cinco anos, passando de $\mathrm{R} \$ 140$ milhões, no ano de 2010, para $\mathrm{R} \$$ 838 milhões, em 2014. Ainda em 2014, a Secretaria Estadual da Saúde de São Paulo gastou R\$ 430 milhões com demandas judiciais dessa ordem ${ }^{(10)}$.

Convém ressaltar que aproximadamente $55 \%$ dos gastos com judicialização da saúde nos municípios do estado de São Paulo foram destinados ao fornecimento de medicamentos, dos quais $45 \%$ não pertenciam aos programas de assistência farmacêutica do SUS ${ }^{(7)}$. Nessa concepção, o direito à saúde acaba por ser resumido à oferta de medicamentos, reduzindo-se a ações curativas que pouco consideram o caráter fundamental de promoção e prevenção em saúde ${ }^{(11-13)}$.

Embora a judicialização represente as reivindicações de cidadãos e instituições em busca da garantia da promoção da saúde e dos direitos de cidadania, esse mecanismo exerce um papel negativo sobre as diretrizes que orientam o SUS ${ }^{(11,14)}$. Estudos que abordam essa temática chamam a atenção, principalmente: para os desequilíbrios orçamentários e as dificuldades da consecução de políticas públicas previstas ${ }^{(7)}$; para o rompimento dos princípios básicos do SUS ${ }^{(3-5,15)}$; para problemas relacionados à garantia de acesso e obtenção de medicamentos ${ }^{(16)}$, e para o aumento das desigualdades ${ }^{(3,17)}$.

A necessidade em compreender e reduzir esse fenômeno emergente impulsiona pesquisadores, gestores e demais atores responsáveis pelos processos decisórios nacionais, tendo em vista o direito universal à saúde e a premissa diretiva do SUS que preconiza uma otimização de recursos. Assim sendo, situa-se a importância de analisar a estreita relação de fatores sociais, econômicos e individuais na busca pelo sistema judiciário ${ }^{(9)}$, bem como as características do aparato em saúde que coexistem com essas ordens judiciais, a fim de demonstrar áreas do SUS que necessitam de mais atenção dos gestores e maior investimento diante das necessidades individuais e 
coletivas de saúde ${ }^{(14)}$. Acrescenta-se, ainda, o ineditismo da presente investigação ao analisar indicadores pouco explorados em estudos dessa natureza.

Desta maneira, o presente estudo tem por objetivo analisar a relação de fatores socioeconômicos e de desempenho do sistema de saúde com a ocorrência de processos judiciais contra o setor público da saúde no Brasil.

\section{MÉTODOS}

Trata-se de um estudo transversal, de abordagem quantitativa, construído por meio de dados secundários de processos judiciais contra o setor público da saúde e de índices socioeconômicos e de desempenho de saúde dos municípios do estado de São Paulo, São Paulo, Brasil.

A amostra analisada foi composta por 641 municípios de SP, agrupados sob 17 Departamentos Regionais de Saúde (DRS), assim como sugerido pelo Sistema de Coordenação das Demandas Estratégicas do SUS (S-Codes). Os dados secundários investigados compreenderam: o Índice Paulista de Responsabilidade Social (IPRS), o Índice de Desempenho do Sistema Único de Saúde (IDSUS) e o Índice de Gini. O período de coleta de dados aconteceu entre julho e novembro de 2015. Os dados têm referência ao ano de 2010 e são provenientes do Ministério da Saúde ${ }^{(18)}$ (base de dados do IDSUS), do S-Codes ${ }^{(19)}$ (base de dados para o número de ações judiciais por DRS), da Fundação Sistema Estadual de Análise de $\operatorname{Dados}^{(20)}$ (Fundação Seade: base de dados para o IPRS) e do Atlas de Desenvolvimento Humano ${ }^{(21)}$ (base de dados para o Índice de Gini).

Os pesquisadores elegeram o ano de 2010 devido à abrangência e à qualidade dos indicadores que compõem o IDSUS desse ano, divulgado em 2012 pelo Ministério da Saúde. Convém destacar que, segundo o Ministério da Saúde, o IDSUS, a princípio, seria divulgado trienalmente, entretanto não houve nova atualização até dezembro de $2017^{(18)}$.

Analisam-se os municípios do estado de São Paulo devido ao elevado volume de ordens judiciais contra o SUS ${ }^{(10)}$, além da viabilidade técnica de acesso aos dados necessários para a condução da presente pesquisa ${ }^{(19)}$. Ressalta-se que o processo de planejamento estadual é voltado a priorizar o papel das instâncias regionais quanto à identificação de problemas de saúde, investimentos e políticas públicas, por isso, a análise é pautada nos DRS. Enfatiza-se também que as ações judiciais consideradas são as ativas, com recibos emitidos, que estão em processo de compra de medicamento.

Para compor o perfil socioeconômico dos municípios de SP, este trabalho empregou o Índice Paulista de Responsabilidade Social (IPRS), um instrumento que avalia o desenvolvimento econômico e social dos municípios paulistas considerando as dimensões de riqueza, longevidade e escolaridade. Este índice estratifica os municípios em cinco grupos, sendo que, no Grupo 1, estão aqueles que se caracterizam por um nível elevado de riqueza, com bons níveis de indicadores sociais, enquanto no Grupo 5 estão os municípios mais desfavorecidos ${ }^{(20)}$. Utilizou-se também o Índice de Gini para medir a desigualdade de distribuição de renda, que pode variar entre 0 e 1 , sendo 0 número 0 para representar municípios de extrema igualdade ${ }^{(21)}$.

Para medir o desempenho do SUS, empregou-se o Índice de Desempenho do Sistema Único de Saúde (IDSUS). Esse indicador fornece uma avaliação global da qualidade do sistema público de saúde brasileiro por meio da análise de acesso potencial aos serviços em saúde e a efetividade das ações prestadas. O IDSUS considera a complexidade do processo demográfico, social e sanitário do país, e é ponderado em Grupos Homogêneos (GH), para uma comparação direta entre municípios com características próximas. A formação desses grupos leva em consideração o Índice de Desenvolvimento Socioeconômico (IDSE), o Índice de Condições de Saúde (ICS) e o Índice de Estrutura do Sistema de Saúde dos Municípios (IESSM). Em termos de classificação, o GH 1 representa o grupo de maior desempenho, enquanto o GH 6 agrupa os municípios com pior desempenho relativo ${ }^{(18)}$.

Estabelecido o conjunto amostral desta pesquisa, dividiu-se em dois níveis de análise, no intuito de alcançar resultados mais robustos ao excluir possíveis outliers. Utilizou-se um método baseado no intervalo interquartil (IIQ), diferença entre o quartil 3 (Q3) e o quartil 1 (Q1). O critério estabelece um limite inferior (LI = Média - 1,5 x IIQ) e um limite superior ( $\left(\mathrm{S}=\right.$ Média $+1,5 \times \mathrm{IIQ}$ ), sendo os valores que extrapolam esses limites considerados outliers ${ }^{(22)}$.

Para a análise empírica, elaborou-se um banco de dado no software Stata (version 14). Inicialmente, utilizouse a matriz de correlação de Pearson para selecionar quais índices comporiam o modelo final de análise. Depois, procedeu-se uma análise de regressão linear múltipla pelo método dos Mínimos Quadrados Ordinários (MQO), em que o número de ações judiciais é explicado por uma relação linear com o desempenho da saúde (IDSUS) e fatores socioeconômicos (Índice de Gini e IPRS), relação representada pelos coeficientes $\beta_{1}, \beta_{2}$ e $\beta_{3}$, respectivamente. $O$ modelo de regressão ainda contempla a constante (c) e o termo de erro aleatório (u). Este último leva em conta 
atributos e elementos não considerados no modelo proposto, enquanto a constante (c) representa o intercepto da reta com o eixo vertical, ou seja, ela independe das variáveis IDSUS, Gini e IPRS do modelo a seguir:

$$
\text { Ações Judiciais }=c+\beta_{1} \text { (IDSUS) }+\beta_{2}\left(\text { GINI) }+\beta_{3}(\text { IPRS })+u\right.
$$

A análise de regressão foi realizada por DRS, com os indicadores sendo ponderados pela população dos municípios. As hipóteses de pesquisa foram testadas estatisticamente, com nível de confiança de $95 \%$ no $p$-valor, mesmo nível considerado para os coeficientes de correlação.

O presente estudo dispensou a apreciação por Comitê de Ética em Pesquisa por se tratar de uma pesquisa com fonte de dados secundários, que não envolveu sujeitos humanos direta ou indiretamente.

\section{RESULTADOS}

No que se refere ao panorama da situação socioeconômica e de saúde dos municípios do estado de São Paulo, na Tabela I está descrita a distribuição do IDSUS estratificado por grupos homogêneos. Observa-se que a maioria dos municípios (62,8\%; n=405) está alocada no chamado Grupo Homogêneo 5 (GH 5), seguido pelo Grupo Homogêneo 3 ( $\mathrm{GH} 3 ; 27,8 \%, n=179)$. Apenas seis municípios integram o Grupo Homogêneo 1 (GH 1), ou seja, municípios que apresentaram as melhores avaliações no atendimento à população, segundo o Ministério da Saúde.

Destaca-se que os municípios estão agrupados por DRS devido às suas características socioeconômicas semelhantes e à proximidade geográfica dos municípios. Em termos relativos, os DRS I, IV e VII apresentaram os melhores resultados do IDSUS em 2010, uma vez que a maioria de seus municípios está alocada nos três primeiros grupos homogêneos, enquanto que o DRS XII tem $87 \%$ dos municípios entre o GH 5 e o GH 6 (Tabela I).

Referente à desigualdade dos municípios, de acordo com os dados do Atlas do Desenvolvimento do Brasil (2010), o Índice de Gini de SP, no ano de 2010, foi de aproximadamente 0,56. O município de Santana de Parnaíba (pertencente ao DRS I - Grande São Paulo) obteve o maior índice ( $\mathrm{GINI}=0,67)$, enquanto o município de Elisiário (contido no DRS XV - São José do Rio Preto) apresentou o menor índice $(G I N I=0,33)$. Para se chegar à média do Gini para cada DRS, o índice foi ponderado pela população dos municípios pertencentes a cada um. Com esse método, o Índice de Gini não apresentou grande diferença de desigualdade entre os DRS $(0,42 \leq \mathrm{GINI} \geq 0,51)$, conforme dados descritos na Tabela I.

Tabela I - Distribuição do Índice de Desempenho do Sistema Único de Saúde (IDSUS) segundo Grupos Homogêneos, e média e desvio padrão do Índice de Gini por Departamentos Regionais de Saúde (DRS). São Paulo, Brasil, 2010.

\begin{tabular}{|c|c|c|c|c|c|c|c|c|c|}
\hline \multirow[b]{2}{*}{ Departamentos Regionais de Saúde (DRS) } & \multicolumn{7}{|c|}{$\begin{array}{c}\text { Número de municípios por Grupos } \\
\text { Homogêneos (GH) do Índice de Desempenho } \\
\text { do Sistema Único de Saúde (IDSUS) }\end{array}$} & \multicolumn{2}{|c|}{ Índice de Gini } \\
\hline & GH1 & GH2 & GH3 & GH4 & GH5 & GH6 & Total & Média & Desvio padrão \\
\hline DRS I - GRANDE SÃO PAULO & 1 & 8 & 17 & 1 & 12 & - & 39 & 0,51 & 0,06 \\
\hline DRS II - ARAÇATUBA & - & 1 & 9 & - & 30 & - & 40 & 0,42 & 0,05 \\
\hline DRS III - ARARAQUARA & - & 2 & 8 & - & 14 & - & 24 & 0,44 & 0,04 \\
\hline DRS IV - BAIXADA SANTISTA & 1 & - & 7 & 1 & - & - & 9 & 0,51 & 0,03 \\
\hline DRS V - BARRETOS & - & 1 & 4 & - & 13 & - & 18 & 0,44 & 0,04 \\
\hline DRS VI - BAURU & - & 3 & 15 & - & 46 & 4 & 68 & 0,45 & 0,05 \\
\hline DRS VII - CAMPINAS & 1 & 1 & 23 & - & 17 & - & 42 & 0,47 & 0,05 \\
\hline DRS VIII - FRANCA & - & 1 & 4 & - & 17 & - & 22 & 0,47 & 0,06 \\
\hline DRS IX - MARÍLIA & - & 1 & 12 & - & 47 & 2 & 62 & 0,45 & 0,05 \\
\hline DRS X - PIRACICABA & - & 2 & 6 & - & 18 & - & 26 & 0,48 & 0,07 \\
\hline DRS XI - PRESIDENTE PRUDENTE & - & 1 & 10 & - & 31 & 3 & 45 & 0,44 & 0,06 \\
\hline DRS XII - REGISTRO & - & - & 2 & - & 7 & 6 & 15 & 0,51 & 0,02 \\
\hline DRS XIII - RIBEIRÃO PRETO & 1 & - & 10 & - & 15 & - & 26 & 0,46 & 0,05 \\
\hline DRS XIV - SÃO JOÃO DA BOA VISTA & - & - & 11 & - & 9 & - & 20 & 0,47 & 0,05 \\
\hline DRS XV - SÃO JOSÉ DO RIO PRETO & 1 & 1 & 11 & - & 89 & - & 102 & 0,42 & 0,05 \\
\hline DRS XVI - SOROCABA & 1 & - & 17 & 1 & 22 & 7 & 48 & 0,48 & 0,05 \\
\hline DRS XVII - TAUBATÉ & - & 2 & 13 & 1 & 18 & 5 & 39 & 0,50 & 0,05 \\
\hline Total de municípios & 6 & 24 & 179 & 4 & 405 & 27 & 645 & 0,46 & 0,06 \\
\hline
\end{tabular}

Fonte: IDSUS, 2010, e Atlas do Desenvolvimento do Brasil, 2010 
Ao se aplicar o método baseado no IIQ sobre os números de processos judiciais por DRS, observou-se que o DRS I - Grande São Paulo apresentou um valor atípico se comparado aos demais, com 8.189 processos judiciais, enquanto a média foi de 1.364 ações (Tabela II). Assim, definiu-se como amostra Nível 1 todos os 17 DRS e amostra Nível 2 o DRS I - Grande São Paulo.

A Tabela II descreve o quantitativo de ações judiciais por DRS no ano de 2010. Observa-se que o maior número de ações judiciais ocorre nos grandes polos urbanos, uma vez que o DRS I possui o maior número de processos (35,5\%), seguido pelo DRS XV (15,9\%). O DRS XII apresentou o menor número de processos judiciais, com um total de sete casos no ano de 2010.

Tabela II - Número de ações judiciais contra o setor público da saúde segundo os Departamentos Regionais de Saúde (DRS). São Paulo, Brasil, 2010.

\begin{tabular}{lcc}
\hline Departamentos Regionais de Saúde (DRS) & Número de Municípios & Número de Processos Judiciais \\
\hline DRS I - GRANDE SÃO PAULO & 39 & 8.189 \\
DRS II - ARAÇATUBA & 40 & 243 \\
DRS III - ARARAQUARA & 24 & 683 \\
DRS IV - BAIXADA SANTISTA & 9 & 689 \\
DRS V - BARRETOS & 18 & 540 \\
DRS VI - BAURU & 68 & 2.235 \\
DRS VII - CAMPINAS & 42 & 1.208 \\
DRS VIII - FRANCA & 22 & 764 \\
DRS IX - MARÍLIA & 62 & 297 \\
DRS X - PIRACICABA & 26 & 764 \\
DRS XI - PRESIDENTE PRUDENTE & 43 & 553 \\
DRS XII - REGISTRO & 15 & 7 \\
DRS XIII - RIBEIRÃO PRETO & 25 & 1.785 \\
DRS XIV - SÃO JOÃO DA BOA VISTA & 20 & 492 \\
DRS XV - SÃO JOSÉ DO RIO PRETO & 102 & 3.679 \\
DRS XVI - SOROCABA & 47 & 616 \\
DRS XVII - TAUBATÉ & 39 & 452 \\
Total Geral & 641 & 23.003 \\
Média & 38 & 1.364 \\
Quartil 1 & 21 & 472 \\
Quartil 3 & 45 & 1.497 \\
Limite Inferior & - & -172 \\
Limite Superior & - & 2.901 \\
\hline
\end{tabular}

Fonte: S-Codes. Nota: A respeito da quantidade de processos judiciais em 2010, os municípios de Guaribá, Itaóca, Marabá Paulista e Martinópolis não contemplaram nenhum DRS porque não havia dados disponíveis desses municípios

Ademais, encontrou-se uma correlação positiva do IDSUS com a incidência de ações judiciais contra o SUS. No Nível 1 houve correlação forte $(p<0,05)$, enquanto que, no Nível 2, houve correlação moderada $(p<0,05)$, conforme pode ser observado na Tabela III.

O Índice de Gini apresentou-se correlacionado de forma moderada e positiva com as ações judiciais no Nível 1 ( $p<0,05)$. Já no Nível 2 , o mesmo índice evidenciou correlação fraca e negativa $(p<0,05)$ (Tabela III). O IPRS apresentou-se pouco representativo $(p>0,05)$ e houve uma correlação quase imperceptível com a variável explicada ${ }^{(22)}$.

Devido a isso, as hipóteses de pesquisa testadas abordam, sobretudo, fatores relacionados ao desempenho dos serviços de saúde do sistema público (IDSUS) e à desigualdade socioeconômica (Índice de Gini). Excluiu-se, então, a variável IPRS do modelo de regressão. 
Tabela III - Matriz de correlação das variáveis socioeconômicas com o número de ações judiciais por nível de Departamentos Regionais de Saúde (DRS). São Paulo, Brasil, 2010.

\begin{tabular}{|c|c|c|c|}
\hline $\begin{array}{l}\text { Ações Judiciais por nível de } \\
\text { Departamentos Regionais de }\end{array}$ & $\begin{array}{c}\text { Índice de Desempenho do Sistema Único } \\
\text { de Saúde (IDSUS) }\end{array}$ & Índice de Gini & $\begin{array}{l}\text { Índice Paulista de } \\
\text { Responsabilidade } \\
\text { Social (IPRS) }\end{array}$ \\
\hline Saúde (DRS) & Coeficiente de correlação & $\begin{array}{l}\text { Coeficiente de } \\
\text { correlação }\end{array}$ & $\begin{array}{l}\text { Coeficiente de } \\
\text { correlação }\end{array}$ \\
\hline $\begin{array}{l}\text { Ações Judiciais no Nível } 1 \\
\text { (p-valor) }\end{array}$ & $\begin{array}{c}0,78 \\
(p<0,05)\end{array}$ & $\begin{array}{c}0,58 \\
(p<0,05)\end{array}$ & $\begin{array}{c}0,10 \\
(p>0,05)\end{array}$ \\
\hline $\begin{array}{l}\text { Ações Judiciais no Nível } 2 \\
\text { (p-valor) }\end{array}$ & $\begin{array}{c}0,55 \\
(p<0,05)\end{array}$ & $\begin{array}{c}-0,35 \\
(p<0,05) \\
\end{array}$ & $\begin{array}{c}0,15 \\
(p>0,05) \\
\end{array}$ \\
\hline
\end{tabular}

Nível 1: todos os DRS; Nível 2: todos os DRS, exceto o DRS I - Grande São Paulo

Quanto aos resultados da regressão linear das variáveis em estudo, pode ser observado na Tabela IV o índice de desempenho na área da saúde (IDSUS) obteve significância estatística com nível de confiança de 95\%. É interessante observar que a relação entre o IDSUS e o número de ações judiciais foi positiva. Com relação ao Índice de Gini, o coeficiente $\beta_{2}$ apresentou relação positiva com as ações judiciais no Nível 1 e relação negativa no Nível 2, no entanto sem significância no p-valor em ambas as amostras, ainda que significante a 10\% no Nível 2.

Tabela IV - Resultados do modelo de regressão linear em que o número de ações judiciais é explicado pelo Índice de Desempenho do Sistema Único de Saúde (IDSUS) e pelo Índice de Gini (GINI). São Paulo, Brasil, 2010.

\begin{tabular}{lcc}
\hline & Variável Dependente & Número de Ações Judiciais \\
\cline { 2 - 3 } & Nível 1 & Nível 2 \\
\hline Constante (c) & $-4,677$ & 4,373 \\
Desvio Padrão & $4.452,00$ & $2.954,52$ \\
p-valor & 0,3113 & 0,1626 \\
IDSUS $\left(\beta_{1}\right)$ & 1,858 & 0,905 \\
Desvio Padrão & $464,199^{*}$ & $308,889^{*}$ \\
p-valor & 0,0013 & 0,0116 \\
GINI $\left(\beta_{2}\right)$ & 2,984 & $-11,134$ \\
Desvio Padrão & $-9,645,47$ & $5.931,59$ \\
p-valor & 0,7600 & 0,0831 \\
$\mathbf{n}^{\circ}$ Observações & 17 & 16 \\
F & 11,00027 & 5,467773 \\
p-valor & 0,001345 & 0,018910 \\
R-Quadrado & 0,611111 & 0,456875 \\
\hline
\end{tabular}

*significância estatística a 5\%. Nível 1: todos os DRS; Nível 2: todos os DRS, exceto o DRS I - Grande São Paulo. n: número

\section{DISCUSSÃO}

A partir da análise do perfil socioeconômico dos municípios avaliados, é possível observar a complexidade dos fatores que podem estar relacionados ao aumento de diligências contra o setor público da saúde. Embora os indicadores socioeconômicos e de saúde do estado de São Paulo estejam acima da média em termos de Brasil (Gini e IDSUS), observa-se que há uma grande diferença entre os municípios representados por DRS.

O principal resultado do presente estudo constatou uma maior demanda judicial entre os municípios com melhor estrutura e prestação de serviço em saúde, contrariando a primeira hipótese formulada $\left(\mathrm{H}_{1}\right)$. Acrescenta-se que essa constatação se manteve nos dois grupos amostrais analisados (Nível 1 e Nível 2), demonstrando relação positiva entre o porte do município e a ocorrência de ações judiciais.

Um estudo recente, que explorou a sensibilidade do IDSUS em relação a variáveis socioeconômicas, identificou que os municípios melhores qualificados quanto ao IDSUS apresentam mais investimento per capita em saúde ${ }^{(23)}$. 
Esses mesmos elementos já haviam sido apontados em estudos anteriores como possíveis responsáveis pelo aumento de demandas judiciais contra o Sistema Único de Saúde ${ }^{(13,24)}$.

A relação entre a judicialização e a avaliação de desempenho do SUS, sobretudo a baixa demanda judicial em municípios com desempenho ruim, pode indicar a necessidade de ações de gestão pública que resultem em melhoria do acesso aos serviços, assim como identificado em estudo que explorou a judicialização para acesso a procedimentos ambulatoriais e hospitalares no estado de Minas Gerais, entre 1999 e $2009^{(24)}$.

No que tange à promoção da saúde, um trabalho com profissionais da área verificou que se posicionam negativamente diante da realidade imposta pela judicialização, mas reconhecem esse recurso como necessário frente à crise na saúde pública ${ }^{(11)}$. Esses resultados ressaltam a imperatividade de ações que estabeleçam a equidade e minimizem os problemas relacionados ao acesso à saúde no Brasil como estratégia de exercício da cidadania, promoção da saúde e maior eficácia das políticas de saúde.

Quanto ao incentivo financeiro, tem-se que os municípios com centros médicos de média e alta complexidade tendem a atrair pessoas de outras localidades por livre demanda ou também por encaminhamento oriundo dos consórcios intermunicipais ${ }^{(13,14)}$. Estudo que avaliou a hipótese de que a judicialização no Brasil seria promovida por elites econômicas identificou uma migração muito elevada quanto à cidade de origem do requerente em relação à cidade de tratamento do mesmo ${ }^{(17)}$. Entretanto, junto com esse novo contingente populacional, os municípios recebem também uma demanda judicial com origem externa, impactando negativamente nos orçamentos públicos e desafiando os gestores municipais no controle e otimização dos recursos ${ }^{(11,25)}$.

Destaca-se que o processo de judicialização para consecução de procedimentos em saúde tem sido maior na população acima dos 50 anos de idade ${ }^{(24)}$, que, juntamente com o envelhecimento populacional, gera uma pressão sobre os gastos públicos ${ }^{(26)}$. Neste sentido, a judicialização não tem considerado a globalidade das políticas públicas, em especial aquelas voltadas à promoção da saúde ${ }^{(27)}$, como que as respostas à demanda por novas tecnologias e por procedimentos de alto custo têm sido mais rápidas do que as ações para a desospitalização ${ }^{(28)}$.

Considerando que, no estado de São Paulo, a taxa média de sucesso das ações contra o setor público da saúde ultrapassa $90 \%{ }^{(19)}$, surge a desconfiança de parcerias entre a indústria farmacêutica, médicos e advogados no processo de judicialização ${ }^{(17,24,29)}$, principalmente nos grandes municípios ou capitais (Nível 1), onde concentram-se os maiores investimentos na área da saúde. Esta maior receptividade do Poder Judiciário em relação às demandas judiciais na saúde recai no fato de que esse sistema deliberativo considera o termo "essencial" em suas decisões como adjetivo equivalente à "indispensável" na garantia do direito à saúde do usuário "(6,24)

Apesar dos resultados do presente estudo demonstrarem que os municípios com piores IDSUS possuem menor número de ações judiciais, um fato a se destacar é que, em municípios de pequeno porte, geralmente os de menores IDSUS, a ocorrência de uma simples ação já é suficiente para comprometer o seu orçamento, prejudicando os investimentos básicos em saúde ${ }^{(7)}$. Este é o caso do município de Buritama, São Paulo, em que, para o ano de 2012, mais da metade do orçamento público na área da saúde foi comprometido com gastos em mandados judiciais ${ }^{(30)}$.

Com base nos resultados encontrados no presente estudo, é evidente a importância de um trabalho intersetorial entre as diversas áreas da gestão pública, bem como o envolvimento de todas as esferas de poder para elaboração e execução de políticas públicas que se adéquem às necessidades da população. Sem dúvidas, a discussão do fenômeno da judicialização passa pela moldura das relações entre poderes estadual, executivo e judiciário, na formulação e execução da política pública de saúde, sobretudo quando o último o faz, predominantemente, na ótica das necessidades individuais, e não coletivas, de saúde.

Há que se salientar um contraponto positivo do fenômeno em questão ao utilizar uma lógica de mercado na gestão de serviços a favor da eficiência do sistema de saúde. Nesse caso, a judicialização passa a ser aliada da saúde pública por sinalizar as deficiências e estimular a reflexão para novas políticas, de forma a reduzir a distância entre o SUS estabelecido no arcabouço normativo e o SUS que executa as ações e serviços de saúde(11,13,31).

Os resultados identificados devem ser analisados com cautela, pois explicam o incremento judiciário da saúde por meio de um número restrito de indicadores. Considerando a natureza multifacetada dos processos de saúde e doença, acredita-se que muitos outros fatores podem contribuir para a incidência desse desfecho, tais como práticas e hábitos em saúde, condições ambientais que propiciam o adoecimento, acesso aos serviços e aos órgãos de justiça, entre outros. Portanto, a vulnerabilidade do IDSUS em retratar características demográficas, sociais e sanitárias apresenta-se como uma limitação do presente estudo, assim como a conveniência de seleção do local de pesquisa, que implica na não generalização dos resultados para outras regiões do país.

Recomenda-se, em pesquisas futuras, uma melhor qualificação dos dados, com estudos que abordem a judicialização da saúde, sobretudo por meio da inclusão de indicadores que expliquem o fenômeno de forma direta 
e que, certamente, subsidiam a discussão, tais como: o perfil sociodemográfico das pessoas e a identificação do tipo de ação judicial e do local de origem do indivíduo proponente.

\section{CONCLUSÃO}

Os resultados apontam que não houve relação linear estatisticamente significativa entre os fatores socioeconômicos analisados (IPRS e Gini) e a ocorrência de ações judiciais contra o setor público da saúde. Contudo, verificou-se um maior número de ações judiciais em localidades com melhor desempenho dos serviços de saúde (IDSUS), ou seja, municípios com melhores IDSUS têm sofrido mais com a judicialização da saúde.

\section{CONFLITOS DE INTERESSE}

Os autores declaram que não há conflitos de interesse no estudo realizado.

\section{CONTRIBUIÇÕES}

Rafael Lutzoff de Camargo Vaz contribuiu com a concepção e desenho do estudo, a coleta, a análise dos dados e a redação inicial. Matheus da Costa Gomes contribuiu com as análises, os procedimentos metodológicos, a interpretação dos resultados e a redação final. Jessica Adrielle Teixeira Santos contribuiu com as análises, a interpretação dos resultados, a redação e aprovação da versão final a ser publicada. Carlos Alberto Grespan Bonacim orientou a pesquisa, contribuiu com as análises, a interpretação dos resultados e a revisão final da versão a ser publicada.

\section{REFERÊNCIAS}

1. Organização das Nações Unidas. Declaração Universal dos Direitos Humanos: declaração de 1948. 2009 [acesso em 2018 Jun 24]. Disponível em: http://www.onu.org.br/img/2014/09/DUDH.pdf

2. Brasil. Constituição Federal de 1988. [acesso em 2016 Maio 22]. Disponível em: http://www.planalto.gov.br/ ccivil_03/constituicao/constituicaocompilado.htm

3. Vieira FS, Zucchi P. Distorções causadas pelas ações judiciais à política de medicamentos no Brasil. Rev Saúde Pública. 2007;41(2):214-22.

4. Vieira FS. Ações judiciais e direito à saúde: reflexão sobre a observância aos princípios do SUS. Rev Saúde Pública. 2008;42(2):365-9.

5. Stival SLM, Girão F. A judicialização da saúde: breves comentários. Cad Ibero-Am Direito Sanit. 2016;5(2):14158.

6. Marques SB, Dallari SG. Garantia do direito social à assistência farmacêutica no Estado de São Paulo. Rev Saúde Pública. 2007;41(1):101-7.

7. Wang DWL, Vasconcelos NP, Oliveira VE, Terrazas FV. Os impactos da judicialização da saúde no município de São Paulo: gasto público e organização federativa. Rev Adm Pública. 2014;48(5):1191-206.

8. Ventura M, Simas L, Pepe VLE, Schramm FR. Judicialização da saúde, acesso à justiça e a efetividade do direito à saúde. Physis (Rio de J). 2010;20(1):7-10.

9. Advocacia Geral da União (BR). Intervenção judicial na saúde pública: panorama no âmbito da Justiça Federal e apontamentos na seara das Justiças Estaduais. 2012 [acesso em 2016 Jan 17]. Disponível em: http://u.saude. gov.br/images/pdf/2014/maio/29/Panorama-da-judicializa----o---2012---modificado-em-junho-de-2013.pdf

10. Cambricoli F. Governos gastam R $\$ 314$ milhões com remédio importado. O Estado de São Paulo [Internet]. 2015 Maio [acesso em 2016 Ago 31]. Disponível em: http://saude.estadao.com.br/noticias/geral,governos-gastam-r314-milhoes-com-remedio-importado, 1693438

11. Ramos RS, Gomes AMT, Oliveira DC, Marques SC, Spindola T, Nogueira VPF. Access the Unified Health System actions and services from the perspective of judicialization. Rev Latinoam Enferm. 2016;24:e2797.

12. Brasil. Lei n. 8.080, de 19 de setembro de 1990. Diário Oficial da União; Brasília; 1990 [acesso em 2016 Jan 13]. Disponível em: http://www.planalto.gov.br/ccivil_03/Leis/L8080.htm 
13. Chieffi AL, Barata RB. Judicialização da política pública de assistência farmacêutica e equidade. Cad Saúde Pública. 2009;25(8):1839-49.

14. Oliveira MRM, Delduque MC, Sousa MF, Mendonça AVM. Judicialização da saúde: para onde caminham as produções científicas? Saúde Debate. 2015;39(105):525-35.

15. Balestra Neto $O$. A jurisprudência dos tribunais superiores e o direito à saúde - Evolução rumo à racionalidade. Rev Direito Sanit. 2015;16(1):87-111.

16. Freitas MKL, Guimarães PBV. A judicialização de políticas públicas no âmbito da saúde relacionada ao fornecimento de medicamentos pelo Estado brasileiro. Rev Jurídica Luso-Brasileira. 2015;1(3):1061-103.

17. Medeiros M, Diniz D, Schwartz IVD. A tese da judicialização da saúde pelas elites: os medicamentos para mucopolissacaridose. Ciênc Saúde Colet. 2013;18(4):1079-88.

18. Ministério da Saúde (BR). Índice de desempenho do Sistema Único de Saúde. 2012 [acesso em 2015 Abr 10]. Disponível em: http://idsus.saude.gov.br/mapas.html

19. Naffah M Filho, Chieff AL, Correa MCMM. S-Codes: um novo sistema de informações sobre ações judiciais da Secretaria de Estado da Saúde de São Paulo. Bepa. 2010;7(84):18-30.

20. Fundação Seade. Índice Paulista de Responsabilidade Social do Estado de São Paulo. 2011 [acesso em 2015 Ago 25]. Disponível em: http://www.seade.gov.br/projetos/iprs/

21. Atlas do Desenvolvimento Humano. Dados do Índice de Gini para os municípios brasileiros em 2010. 2013 [acesso em 2015 Mar 20]. Disponível em: http://pt.scribd.com/doc/50786275/Atlas-do-Desenvolvimento-Humano

22. Fávero LPL. Análise de dados: modelos de regressão com Excel®, Stata® e SPSS $®$. Rio de Janeiro: Elsevier; 2015.

23. Silva RAG. Sensibilidade do Índice de Desenvolvimento do Sistema Único de Saúde (IDSUS) a variáveis socioeconômicas municipais [dissertação]. Maceió: Universidade Federal de Alagoas; 2015.

24. Gomes FFC, Cherchiglia ML, Machado CD, Santos VC, Acurcio FA, et al. Acesso aos procedimentos de média e alta complexidade no Sistema Único de Saúde: uma questão de judicialização. Cad Saúde Pública. 2014;30(1):31-43.

25. Silva JB, Jucatelli JP. Judicialização da saúde, ativismo judicial e o consequente desequilíbrio do orçamento público. Rev Bras Polít Públicas [Online]. 2017 [acesso em 2015 Mar 20].;7(1):98-115. Disponível em: https:// www.publicacoesacademicas.uniceub.br/RBPP/article/view/4454

26. Carneiro L. O futuro da saúde suplementar. Foco Gestão. 2017,1(1):4-9.

27. Wang $D$. Judicialização da saúde: o aumento no número de ações judiciais representa um risco para a saúde pública e privada. Foco Gestão. 2017,1(1):13-17.

28. Campos GWS, Amaral MAA. A clínica ampliada e compartilhada, a gestão democrática e redes de atenção como referências teórico-operacionais para a reforma do hospital. Ciênc Saúde Colet. 2007;12(4):849-59.

29. Catanheide ID, Lisboa ES, Souza LEPF. Características da judicialização do acesso a medicamentos no Brasil: uma revisão sistemática. Physis (Rio de J). 2016;26(4):1335-56.

30. Oliveira VE, Noronha L. Judiciary-Executive relations in policy making: the case of drug distribution in the State of São Paulo. Bras Political Sci Rev. 2011;5(2):10-38.

31. Wang DWL, Ferraz OLM. Reaching out to the needy? Access to justice and public attorneys' role in right to health litigation in the city of São Paulo. SUR. 2013;10(18):33.

\section{Endereço para correspondência:}

Rafael Lutzoff de Camargo Vaz

Universidade de São Paulo - USP

Faculdade de Economia, Administração e Contabilidade (FEA - RP)

Departamento de Contabilidade

Av. Bandeirantes, 3900

Bairro: Monte Alegre

CEP: 14040-900 - Ribeirão Preto - SP - Brasil

E-mail: rafael.Icvaz@gmail.com 